hep-ph/9805291

$\operatorname{SINP} / \mathrm{TNP} / 98 / 11$

\title{
Gravitational coupling of neutrinos in a medium
}

\author{
José F. Nieves \\ Department of Physics, P.O. Box 23343 \\ University of Puerto Rico, Río Piedras \\ Puerto Rico 00931-3343 \\ Palash B. Pal \\ Saha Institute of Nuclear Physics, 1/AF Bidhan-Nagar \\ Calcutta 700064, India
}

May 1998

\begin{abstract}
In a medium that contains electrons but not the other charged leptons, such as normal matter, the gravitational interactions of neutrinos are not the same for all the neutrino flavors. We calculate the leading order matter-induced corrections to the neutrino gravitational interactions in such a medium and consider some of their physical implications.
\end{abstract}

\section{Introduction}

When neutrinos propagate through a medium, the effects of the background particles can influence their properties in important ways. The possible consequences of these effects have been the subject of continuous research activity in recent years, largely motivated by their implications in a variety of astrophysical and cosmological contexts. Some examples of the latter include the original MSW mechanism [1] and its variations in the context of the Solar Neutrino Problem [2], and the explanation of the large birth velocities of pulsars in terms of the asymmetric emission of neutrinos from the cooling protostar, which is produced by the matter-enhanced neutrino oscillations biased by the supernova's magnetic field [3].

Among the various approaches that exist to the study of the matter effects on the propagation of neutrinos, the methods of Quantum Statistical Field Theory (QSFT) have proven to be very useful ones. These methods have been applied to reproduce the Wolfenstein formula for the index of refraction of neutrinos in matter and to obtain corrections to it 目, 5, 6, 6. In addition, they have been used to determine the electromagnetic properties of a neutrino that propagates in matter by means of the one-loop calculation of the electromagnetic vertex function induced by the neutrino interactions with the background particles [8]. Furthermore, it was observed in Ref. [B] that, in the presence of a static magnetic field, the effective electromagnetic interactions of the neutrinos produce an additional contribution to the neutrino index of refraction which modifies the condition for resonant oscillations in matter. This effect is in fact the origin of the possible explanation of the large birth velocities of pulsars mentioned above.

In all these situations, a common theme has been the observation that the universality of the neutrino interactions is broken due to the fact that normal matter contains electrons but not the other charged leptons. Therefore, in a medium such as the Sun or a supernova, the electron neutrinos on one hand, and the tau and muon neutrinos on the other, are affected in different ways. This fact implies that in a such medium the GIM mechanism is not operative, and it explains why the radiative decay of a neutrino in a medium is greatly enhanced compared to the corresponding rate in the vacuum [10].

The observation that the universality of the neutrino interactions is broken by the background medium is a special case of a more general concept. It is the notion that while the fundamental Lagrangian of the theory is invariant under certain symmetry operations, a given background medium may not be invariant under 
the same operations. Thus, for example, normal matter is neither $C P$ nor $C P T$ asymmetric, a fact that has interesting consequences for the propagation of photons in a (chiral) medium such as a gas of neutrinos [11]. Taking this notion to the extreme, we can even think of the medium as breaking Lorentz invariance since it specifies a preferred frame of reference; i.e., that in which the medium is at rest. In the context of QSFT, this breakdown of Lorentz invariance is traded by an explicit dependence of the calculated physical properties of the particles on the velocity four-vector of the background medium. In this way, for example, the photon acquires a gauge invariant mass, a left-handed neutrino acquires a chiral-invariant mass and a Majorana neutrino acquires electromagnetic dipole moments.

The main observation of the present paper is that, in the presence of medium, the breakdown of the universality of the neutrino interactions includes their gravitational interactions as well. In analogy with the fact that, in a medium that contains electrons but no muons or taus, the electron neutrinos have different electromagnetic interactions than the muon or tau neutrinos, their gravitational interactions also differ. In this work we determine the effective gravitational interactions of neutrinos in a matter background, by calculating the one-loop contribution to the neutrino stress-energy tensor, which is the gravitational analog of the electromagnetic current.

We wish to clarify at the outset the following point. The possibility that the observed deficit of solar neutrinos can be ascribed to neutrino oscillations that are driven by flavor changing interactions of gravitational origin has been proposed previously [12]. In these works, it is postulated that the gravitational interaction of neutrinos has the same form as the standard one, but with different coupling strengths for each neutrino flavor. This assumption violates the equivalence principle in a fundamental way, and in fact destroys the symmetry that makes it consistent to assume the existence of a massless graviton in the vacuum. On the other hand, no such fundamental breaking of the equivalence principle is assumed in the effect that we describe in the present work. Our calculations are based on the standard model of particle interactions, together with the commonly accepted linearized gravitational coupling of fermions via the stress-energy tensor, with a universal coupling strength. The non-universal character of the effective gravitational coupling of the neutrinos, whose calculation is the aim of this work, is a consequence of the flavor asymmetry of the medium and not of the fundamental Lagrangian, as we have emphasized above.

We have found convenient to organize the presentation in the following manner. In Section 2, we outline the linearized theory of the gravitational coupling of fermions, including the interaction terms with the $W$ and $Z$ gauge bosons. This has been necessary because the formulas that are commonly used and/or quoted in the literature are valid when the fermions that couple to the graviton are on their mass-shell. Therefore, it is not appropriate to use them for the internal fermion lines in the one-loop diagrams that we need to consider. Armed with the preliminaries of Section 2, the one-loop diagrams for the induced gravitational interactions of the neutrinos in a background composed of electrons and nucleons are calculated in Section 3 . There we obtain the effective gravitational vertex function as an integral over the momentum distribution functions of the background particles. We conclude that section with some general remarks about the consistency of the one-loop formulas, and in particular we show explicitly that our result for the effective gravitational vertex of the neutrinos, including the background-induced terms, is transverse as it should be. Using the one-loop formulas for the vertex function as a starting point, we determine in Section 4 the modification to the neutrino index of refraction in a medium in the presence of a static gravitational field. There we consider also some possible applications of these results, giving some of the details in the Appendix, and Section 5 contains our conclusions.

\section{Tree-level gravitational couplings}

It is well known that the linear, or weak field, approximation to the metric theory of gravity is equivalent to a quantum particle description of gravitational interactions in which the graviton emerges as a spin- 2 quantum field coupled to the stress-energy tensor. The formulas for the gravitational vertices is given and discussed in many textbooks, at least for the common cases of spin 0,1/2, and spin-1 (i.e., photons) particles. However, the formulas that are customarily quoted are given for the case in which the particles are on their mass-shell, which is sufficient for the applications that have been considered in the literature, but not for the present one. This is particularly true for the fermions.

In the one-loop calculation that we are considering, there are diagrams in which the graviton couples to internal electron lines in a loop. For them, the on-shell form for the gravitational vertex is not valid. 
Furthermore, there are other interaction vertices that are unique to our case in hand. In this section we consider all the couplings that relevant to our calculation in detail. We define the action $\mathcal{A}$ in presence of gravitation by

$$
\mathcal{A}=\int d^{4} x \mathcal{L}
$$

and then look at the different terms in $\mathcal{L}$ which will be relevant for us.

\subsection{Fermion couplings}

The Lagrangian for a free Dirac fermion of mass $m_{f}$ can be written in the explicitly Hermitian form

$$
\mathcal{L}_{0}^{(f)}=\left[\frac{i}{2} \bar{\psi} \gamma^{\mu} \partial_{\mu} \psi+\text { h.c. }\right]-m_{f} \bar{\psi} \psi \text {. }
$$

The Lagrangian in the presence of gravitational interactions is obtained from this by making the replacement $\gamma^{\mu} \partial_{\mu} \rightarrow \gamma^{a} \mathcal{D}_{a}$, where the $\gamma^{a}$ denote the ordinary gamma matrices and

$$
\mathcal{D}_{a}=v_{a}^{\mu}\left(\partial_{\mu}-\frac{i}{4} \omega_{b c \mu} \sigma^{b c}\right)
$$

is the gravity-covariant derivative, with $\sigma^{a b}=\frac{i}{2}\left[\gamma^{a}, \gamma^{b}\right]$, and $v_{a}{ }^{\mu}$ being the vierbein vector fields. These are defined in a way that

$$
\eta^{a b} v_{a}^{\mu} v_{b}^{\nu}=g^{\mu \nu}, \quad g_{\mu \nu} v_{a}^{\mu} v_{b}^{\nu}=\eta_{a b},
$$

where $g_{\mu \nu}$ is the space-time metric and $\eta_{a b}$ is the flat space metric, which we take to be $\operatorname{diag}(1,-1,-1,-1)$. The spin connection coefficients are given by

$$
\omega_{a b \gamma}=v_{a \lambda}\left(\partial_{\gamma} v_{b}^{\lambda}+\Gamma_{\mu \gamma}^{\lambda} v_{b}^{\mu}\right),
$$

where $\Gamma_{\lambda \mu \gamma}$ are the Christoffel symbols. This formula can be expressed explicitly in terms of the vierbein fields in the form

$$
\omega_{a b \gamma}=\frac{1}{2}\left\{v_{a}^{\mu}\left(\partial_{\gamma} v_{b \mu}-\partial_{\mu} v_{b \gamma}\right)+v_{b}{ }^{\mu}\left(\partial_{\mu} v_{a \gamma}-\partial_{\gamma} v_{a \mu}\right)+v_{a}{ }^{\mu} v_{b}{ }^{\nu} v_{c \gamma}\left(\partial_{\nu} v^{c}{ }_{\mu}-\partial_{\mu} v_{\nu}^{c}\right)\right\} .
$$

In addition to these changes, we have to include the determinant of the matrix $v_{a \mu}$, which we denote by $\operatorname{det}(v)$, as an overall factor.

Thus, in the absence of all interactions except the gravitational ones, the Dirac Lagrangian is given by

$$
\mathcal{L}_{g}^{(f)}=\operatorname{det}(v)\left\{\left[\frac{i}{2} \bar{\psi} \gamma^{a} v_{a}{ }^{\mu}\left(\partial_{\mu}-\frac{i}{4} \omega_{b c \mu} \sigma^{b c}\right) \psi+\text { h.c. }\right]-m_{f} \bar{\psi} \psi\right\}
$$

With this construction, the term in the action corresponding to $\mathcal{L}_{g}^{(f)}$ is invariant under general coordinate transformations, labeled by the greek indices, and also under local Lorentz transformations labeled by the latin indices.

The linear theory of the gravitational couplings is obtained by assuming that, for weak gravitational fields, we can write

$$
g_{\mu \nu}=\eta_{\mu \nu}+2 \kappa h_{\mu \nu}
$$

and treat the second term on the right side as a perturbation. The quantity $\kappa$ is related to the Newton's constant $G$ through the equation

$$
\kappa=\sqrt{8 \pi G},
$$

in order that $h_{\mu \nu}$, identified with the graviton quantum field, has the correctly normalized kinetic energy term in the Lagrangian. The vierbeins cannot be determined uniquely from Eq. (2.4). They can be determined 
only up to a local Lorentz transformation which, however, would leave the action invariant. Therefore, we can choose in particular

$$
v_{a \mu}=\eta_{a \mu}+\kappa h_{a \mu},
$$

which in turn gives

$$
\operatorname{det}(v)=1+\kappa \eta_{\mu \nu} h^{\mu \nu}
$$

Note that this also implies that

$$
g^{\mu \nu} \simeq \eta^{\mu \nu}-2 \kappa h^{\mu \nu}
$$

since the matrix $g^{\mu \nu}$ is the inverse of the metric $g_{\mu \nu}$ and from Eq. (2.4),

$$
v_{a}{ }^{\mu}=\eta_{a}{ }^{\mu}-\kappa h_{a}{ }^{\mu} .
$$

We now substitute these relations into Eq. (2.7), and keep only the terms that are at most linear in $\kappa$. Once this approximation is made, it is no longer necessary to distinguish between greek and latin indices (since any difference between them would contribute only to higher order in $\kappa$ ) and therefore we write everything in terms of the greek indices from now on. From Eq. (2.6) we obtain

$$
\omega_{\mu \nu \gamma}=\frac{1}{2}\left(\partial_{\nu} h_{\mu \gamma}-\partial_{\mu} h_{\gamma \nu}\right)
$$

and it then follows that the term involving the $\sigma$-matrices in Eq. (2.7) drops out because it is proportional to the quantity

$$
\left\{\gamma_{\mu}, \sigma_{\lambda \nu}\right\}+(\mu \leftrightarrow \nu)=0 .
$$

From the other terms we then obtain the gravitational interaction Lagrangian of the fermion in the form

$$
\mathcal{L}_{h}^{(f f)}=-\kappa h^{\mu \nu}(x) \widehat{T}_{\mu \nu}^{(f)}(x),
$$

where the stress-energy tensor operator $\widehat{T}_{\mu \nu}^{(f)}$ for the fermion field is given by

$$
\widehat{T}_{\mu \nu}^{(f)}(x)=\left\{\frac{i}{4} \bar{\psi}(x)\left[\gamma_{\mu} \partial_{\nu}+\gamma_{\nu} \partial_{\mu}\right] \psi(x)+\text { h.c. }\right\}-\eta_{\mu \nu} \mathcal{L}_{0}^{(f)}(x) .
$$

From Eqs. (2.16) and (2.17) it follows that the term corresponding to the gravitational fermion vertex in a Feynman diagram is $-i \kappa V_{\mu \nu}^{(f)}$, where

$$
V_{\mu \nu}^{(f)}\left(p, p^{\prime}\right)=\frac{1}{4}\left[\gamma_{\mu}\left(p+p^{\prime}\right)_{\nu}+\gamma_{\nu}\left(p+p^{\prime}\right)_{\mu}\right]-\frac{1}{2} \eta_{\mu \nu}\left[\left(\not p-m_{f}\right)+\left(\not p^{\prime}-m_{f}\right)\right]
$$

In the above considerations we have assumed that the fermion $f$ is a Dirac particle with a given mass. On the other hand, it is easy to infer by inspection that similar arguments yield the formula

$$
V_{\mu \nu}^{(\nu)}\left(k, k^{\prime}\right)=\frac{1}{4}\left[\gamma_{\mu}\left(k+k^{\prime}\right)_{\nu}+\gamma_{\nu}\left(k+k^{\prime}\right)_{\mu}\right] L-\frac{1}{2} \eta_{\mu \nu}\left[\not k+\not k^{\prime}\right] L
$$

for the case of a chiral, left-handed neutrino.

Notice that if we take the matrix element of the operator $\widehat{T}_{\mu \nu}^{(f)}(0)$ between on-shell fermion states, with incoming and outgoing momenta $p$ and $p^{\prime}$ respectively, the term proportional to $\eta_{\mu \nu}$ in Eq. (2.17) gives no contribution and we obtain

$$
\left\langle f\left(p^{\prime}\right)\left|\widehat{T}_{\mu \nu}^{(f)}(0)\right| f(p)\right\rangle=\frac{1}{4} \bar{u}\left(p^{\prime}\right)\left\{\gamma_{\mu}\left(p+p^{\prime}\right)_{\nu}+\gamma_{\nu}\left(p+p^{\prime}\right)_{\mu}\right\} u(p),
$$

which is the expression that is quoted in textbooks [14]. However, for the purposes of the one-loop calculation that we carry out in Section 3, we need to use the vertex for off-shell fermions given in Eq. (2.18). In particular, as we will show in Section 3 , the one-loop calculation of the effective gravitational vertex of the neutrino gives a result that satisfies the transversality condition provided that the term proportional to $\eta_{\mu \nu}$ in Eq. (2.18) is included in the calculation of the loop diagrams. 


\section{$2.2 \quad$ Fermion and $W$ boson couplings}

The interactions that drive the effective gravitational vertex of the neutrinos, are the standard weak interactions with the particles of the background. Let us consider the charged-current interactions first. In the presence of a gravitational field, the interaction term $\bar{e}_{L} \gamma^{\mu} W_{\mu} \nu_{L}$ in the Lagrangian is modified according to

$$
\mathcal{L}_{g}^{(\mathrm{cc})}=\operatorname{det}(v)\left\{-\frac{g}{\sqrt{2}} \bar{e}_{L} \gamma^{a} \nu_{L} v_{a \mu} W^{\mu}+\text { h.c. }\right\} .
$$

In the linear approximation given by Eqs. (2.10) and (2.11), this becomes

$$
\mathcal{L}_{g}^{(\mathrm{cc})}=\mathcal{L}_{0}^{(\mathrm{cc})}+\mathcal{L}_{h}^{(\mathrm{cc})},
$$

where $\mathcal{L}_{0}^{(\mathrm{cc})}$ is the standard charged-current interaction Lagrangian

$$
\mathcal{L}_{0}^{(\mathrm{cc})}=-\frac{g}{\sqrt{2}} W^{\mu} \bar{e}_{L} \gamma_{\mu} \nu_{L}+\text { h.c. }
$$

while

$$
\begin{aligned}
\mathcal{L}_{h}^{(\mathrm{cc})} & =-\kappa \frac{g}{\sqrt{2}} h^{\mu \nu}\left[\eta_{\mu \nu} W_{\alpha} \bar{e}_{L} \gamma^{\alpha} \nu_{L}+\frac{1}{2}\left(W_{\mu} \bar{e}_{L} \gamma_{\nu} \nu_{L}+W_{\nu} \bar{e}_{L} \gamma_{\mu} \nu_{L}\right)+\text { h.c. }\right] \\
& =-\kappa \frac{g}{\sqrt{2}} a_{\mu \nu \lambda \rho} \bar{e}_{L} \gamma^{\lambda} \nu_{L} W^{\rho} h^{\mu \nu}+\text { h.c. },
\end{aligned}
$$

where

$$
a_{\mu \nu \lambda \rho}=\eta_{\mu \nu} \eta_{\lambda \rho}+\frac{1}{2}\left[\eta_{\mu \lambda} \eta_{\nu \rho}+\eta_{\mu \rho} \eta_{\nu \lambda}\right]
$$

\section{$2.3 \quad W$ boson couplings}

The gravitational vertex for photons is well known. For the $W$ boson, the only difference comes from the mass term in the kinetic energy part of the Lagrangian. Indeed, from the usual expression for the kinetic energy of the $W$ vector boson,

$$
\mathcal{L}_{g}^{(W)}=\operatorname{det}(v)\left\{-\frac{1}{2} W_{\mu \nu}^{*} W^{\mu \nu}-M_{W}^{2} W_{\mu}^{*} W^{\mu}\right\},
$$

and then making the substitutions given in Eqs. (2.8) and (2.11), we arrive at

$$
\mathcal{L}_{g}^{(W)}=\mathcal{L}^{(W)}+\mathcal{L}_{h}^{(W W)},
$$

where $\mathcal{L}^{(W)}$ is the standard form of the kinetic energy for the free $W$ boson, while

$$
\mathcal{L}_{h}^{(W W)}=-\kappa h^{\mu \nu}\left[\left(\frac{1}{2} W_{\alpha \beta}^{*} W^{\alpha \beta}-M_{W}^{2} W_{\alpha}^{*} W^{\alpha}\right) \eta_{\mu \nu}+\left(W_{\mu \alpha}^{*} W_{\nu}^{\alpha}-M_{W}^{2} W_{\mu}^{*} W_{\nu}+\text { h.c. }\right)\right]
$$

gives the gravitational vertex.

Of particular importance for us are the terms proportional to the $W$ mass. The reason is the following. In the analogous calculation of the charged-current contribution to the electromagnetic neutrino vertex in a medium, the dominant term is of order $1 / M_{W}^{2}$ and it arises from the diagram that corresponds to diagram (A) in Fig. 1, in which the photon couples to the electron line in the loop. The diagram in which the photon couples to the internal $W$ line, corresponding to diagram (B), is of order $1 / M_{W}^{4}$, and therefore it is negligible. In the present case, because of the presence of the term that is proportional to the $W$ mass in the coupling of the $W$ to the graviton, diagram (B) gives a contribution also of order $1 / M_{W}^{2}$ that must be taken into account. As far as these terms only are concerned, we can then replace Eq. (2.28) by

$$
\mathcal{L}_{h}^{(W W)}=\kappa M_{W}^{2} a_{\mu \nu \lambda \rho}^{\prime} h^{\mu \nu} W^{\lambda} W^{* \rho},
$$

where

$$
a_{\mu \nu \lambda \rho}^{\prime}=\eta_{\mu \nu} \eta_{\lambda \rho}+\eta_{\mu \lambda} \eta_{\nu \rho}+\eta_{\mu \rho} \eta_{\nu \lambda} .
$$



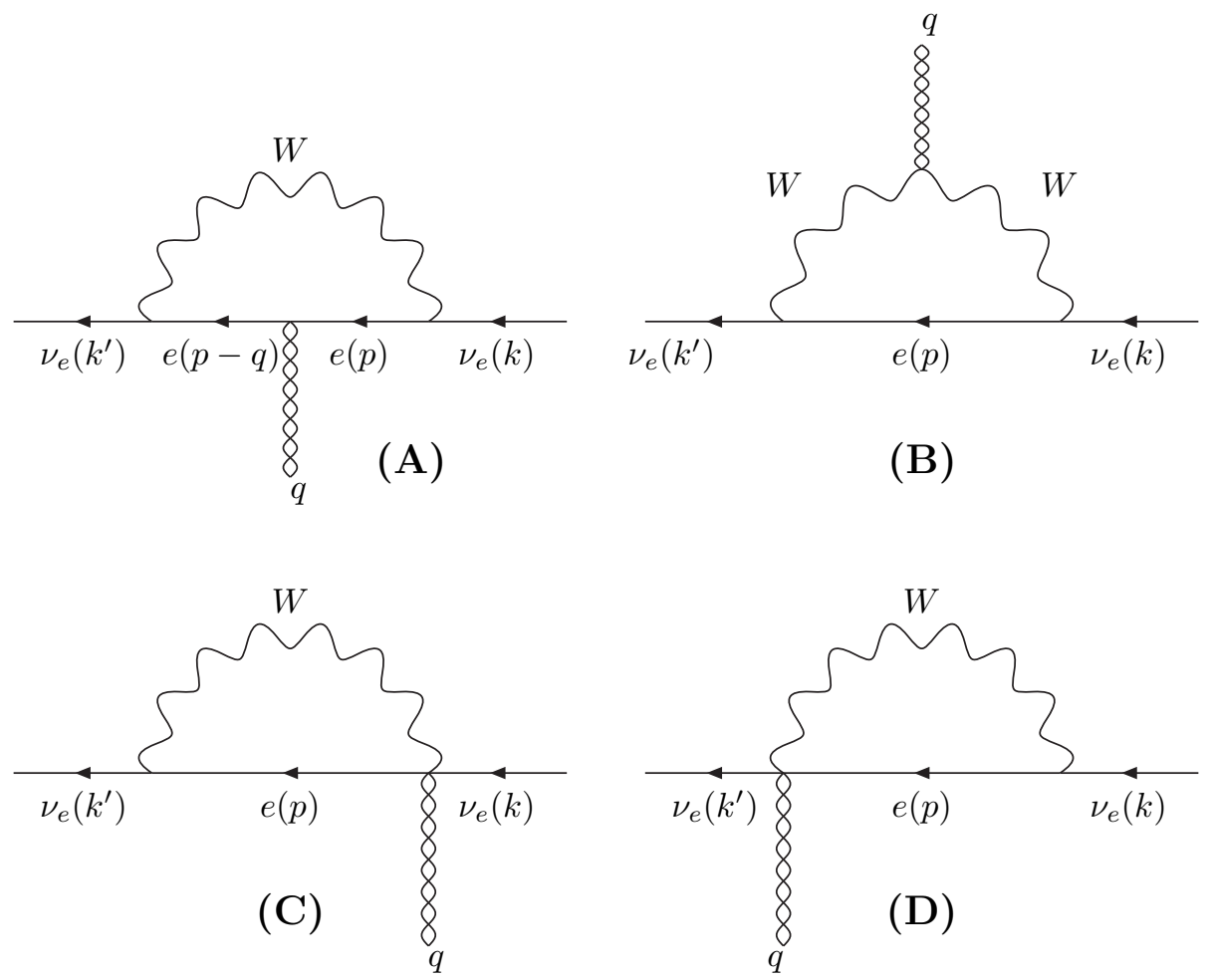

Figure 1: $W$ exchange diagrams for the one-loop contribution to the $\nu_{e}$ gravitational vertex in a background of electrons. The braided line represents the graviton.

\subsection{Including the neutral-current couplings}

The charged current interaction discussed above are relevant only for the $\nu_{e}$ 's. The $\nu_{\mu}$ 's and the $\nu_{\tau}$ 's will interact with the electrons only through the neutral current. Moreover, a normal background contains nucleons as well, which interact with all neutrinos via the neutral current. To take these interactions into account, we consider the neutral current couplings of the $Z$-boson. In absence of gravitation, these are

$$
\mathcal{L}_{0}^{(\mathrm{nc})}=-g_{Z} Z^{\mu}\left[\sum_{i=e, \mu, \tau} \bar{\nu}_{i L} \gamma_{\mu} \nu_{i L}+\sum_{f=e, p, n} \bar{f} \gamma_{\mu}\left(X_{f}+Y_{f} \gamma_{5}\right) f\right],
$$

where, in the standard model,

$$
\begin{aligned}
g_{Z} & =g /\left(2 \cos \theta_{W}\right) \\
-X_{e}=X_{p} & =\frac{1}{2}-2 \sin ^{2} \theta_{W} \\
X_{n} & =-\frac{1}{2} \\
Y_{e} & =\frac{1}{2} \\
Y_{n}=-Y_{p} & =\frac{1}{2} g_{A}
\end{aligned}
$$

with $g_{A}=1.26$ being the renormalization constant of the axial-vector current of the nucleon. It is also useful to remember that, in the standard model,

$$
\frac{g_{Z}^{2}}{M_{Z}^{2}}=\frac{g^{2}}{4 M_{W}^{2}}=\sqrt{2} G_{F}
$$




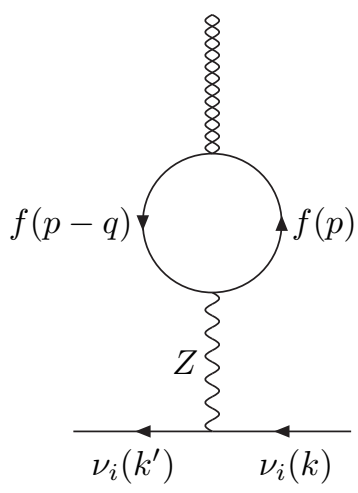

(A)

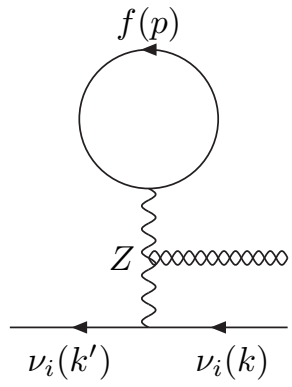

(B)

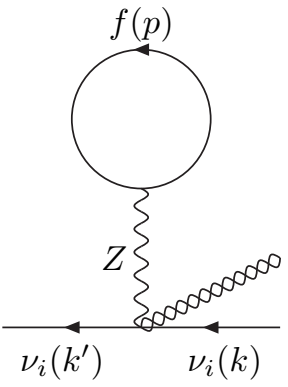

(C)

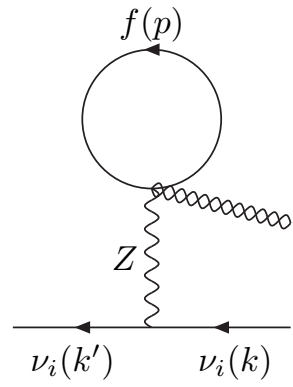

(D)

Figure 2: $Z$-exchange diagrams for the one-loop contribution to the gravitational vertex of any neutrino flavor $(i=e, \mu, \tau)$ in a background of electrons and nucleons.

Following the arguments that were used for the charged current, we find that in presence of gravitation, these interactions will be modified to

$$
\mathcal{L}_{g}^{(\mathrm{nc})}=\mathcal{L}_{0}^{(\mathrm{nc})}+\mathcal{L}_{h}^{(\mathrm{nc})}
$$

where $\mathcal{L}_{0}^{(\mathrm{nc})}$ has been given above in Eq. (2.31), and

$$
\mathcal{L}_{h}^{(\mathrm{nc})}=-\kappa g_{Z} a_{\mu \nu \lambda \rho}\left[\sum_{i=e, \mu, \tau} \bar{\nu}_{i L} \gamma^{\lambda} \nu_{i L}+\sum_{f=e, p, n} \bar{f} \gamma^{\lambda}\left(X_{f}+Y_{f} \gamma_{5}\right) f\right] Z^{\rho} h^{\mu \nu}
$$

where $a_{\mu \nu \lambda \rho}$ has been defined in Eq. (2.25). Also, there are terms which comes from the Lagrangian of the pure $Z$-boson which give interactions with the graviton and are proportional to $M_{Z}^{2}$. These are

$$
\mathcal{L}_{h}^{(Z Z)}=\frac{1}{2} \kappa M_{Z}^{2} a_{\mu \nu \lambda \rho}^{\prime} h^{\mu \nu} Z^{\lambda} Z^{\rho}
$$

where $a_{\mu \nu \lambda \rho}^{\prime}$ is the tensor defined in Eq. (2.30).

\section{Induced gravitational vertex of neutrinos}

We now consider the background contributions to the neutrino gravitational vertex. We denote the proper vertex function for off-shell neutrinos, including the background-induced contributions, by $\Gamma_{\mu \nu}^{(\nu)}\left(k, k^{\prime}\right)$. It is is defined such that the matrix element of the total stress-energy tensor operator $\widehat{T}_{\mu \nu}(x)$, between incoming and outgoing neutrino states with momenta $k$ and $k^{\prime}$ respectively, is given by

$$
\left\langle\nu\left(k^{\prime}\right)\left|\widehat{T}_{\mu \nu}(0)\right| \nu(k)\right\rangle=\bar{u}_{L}\left(k^{\prime}\right) \Gamma_{\mu \nu}^{(\nu)}\left(k, k^{\prime}\right) u_{L}(k)
$$

It is useful to divide the relevant one-loop diagrams into the two sets given in Figs. 1 and 2, according to whether they involve the $W$ or the $Z$ boson. In the diagrams, and in the formulas that follow, $q$ stands for the outgoing graviton momentum

$$
q=k-k^{\prime}
$$

The additional diagrams in which the graviton line comes out from one of the external neutrino legs are not shown, since they are 1-particle reducible and do not contribute to $\Gamma_{\mu \nu}^{(\nu)}$. The proper way to take them 
into account in the calculation of the amplitude for any given process, is by choosing the external neutrino spinor $u_{L}(k)$ to be the (properly normalized) solution of the effective Dirac equation for the propagating neutrino mode in the medium, instead of the spinor representing the free-particle solution of the equation in the vacuum. This will be discussed in more detail in Section 3.4.

As commented earlier, the $W$-exchange diagrams of Fig. 1 contribute only to the gravitational coupling of $\nu_{e}$. In contrast, the diagrams shown in Fig. 2 contribute equally to the gravitational vertex of all the neutrino flavors $\nu_{e, \mu, \tau}$, and therefore are not relevant in phenomena that involve transitions between the standard, weak SU(2)-doublet neutrinos. However, in processes in which the so-called sterile neutrinos participate, these flavor-diagonal contributions are important. Moreover, we consider them first since the results for the $W$-diagrams can be easily obtained from the corresponding ones for the $Z$-diagrams by making some simple substitutions.

\subsection{The $Z$-mediated diagrams}

\subsubsection{Diagram (A)}

This contribution, which will be marked by the superscript $2 \mathrm{~A}$, is given by

$$
-i \kappa \Gamma_{\mu \nu}^{(2 A)}=-\left(-i g_{Z}\right)^{2}(-i \kappa) \frac{i}{M_{Z}^{2}} \gamma^{\lambda} L \sum_{f=e, p, n} \int \frac{d^{4} p}{(2 \pi)^{4}} \operatorname{Tr}\left[\gamma_{\lambda}\left(X_{f}+Y_{f} \gamma_{5}\right) i S_{F}^{(f)}\left(p^{\prime}\right) V_{\mu \nu}^{(f)}\left(p, p^{\prime}\right) i S_{F}^{(f)}(p)\right]
$$

or equivalently

$$
\Gamma_{\mu \nu}^{(2 A)}=\frac{i g_{Z}^{2}}{M_{Z}^{2}} \gamma^{\lambda} L \sum_{f=e, p, n} \int \frac{d^{4} p}{(2 \pi)^{4}} \operatorname{Tr}\left[\gamma_{\lambda}\left(X_{f}+Y_{f} \gamma_{5}\right) i S_{F}^{(f)}\left(p^{\prime}\right) V_{\mu \nu}^{(f)}\left(p, p^{\prime}\right) i S_{F}^{(f)}(p)\right],
$$

In these expressions we have introduced the shorthand

$$
p^{\prime} \equiv p-q,
$$

which will be used throughout the calculations, and we have neglected the momentum dependence of the $Z$ propagator in anticipation of the fact that we are interested only in the terms that are linear in the Fermi constant $G_{F}$. Further, $S_{F}^{(f)}$ is the thermal fermion propagator which is given by

$$
i S_{F}^{(f)}(p)=\left(\not p+m_{f}\right)\left[\frac{i}{p^{2}-m_{f}^{2}+i \epsilon}-2 \pi \delta\left(p^{2}-m_{f}^{2}\right) \eta_{f}(p)\right],
$$

where

$$
\eta_{f}(p)=\frac{\theta(p \cdot v)}{e^{\beta\left(p \cdot v-\mu_{f}\right)}+1}+\frac{\theta(-p \cdot v)}{e^{-\beta\left(p \cdot v+\mu_{f}\right)}+1}
$$

with $\beta$ being the inverse temperature, $\mu_{f}$ the chemical potential and $v^{\mu}$ the velocity four-vector of the medium [15]. In the frame in which the medium is at rest, $v^{\mu}$ has components

$$
v^{\mu}=(1, \mathbf{0}) \text {. }
$$

When the expressions for $S_{F}^{(f)}(p)$ and $S_{F}^{(f)}\left(p^{\prime}\right)$ given Eq. (3.6) are substituted in Eq. (3.4), three kinds of term are generated, according to whether they contain none, one or two factors of $\eta_{f}$. We are interested in the terms with at least one factor of $\eta_{f}$, since that is where the background dependence of the neutrino gravitational vertex will come from. However, the terms with two factors of $\eta_{f}$ are relevant only in the calculation of the absorptive part of the amplitude, which we are not considering here, and therefore the important terms for us are the ones having a single factor of $\eta_{f}$. Denoting their contribution to the vertex function by $\Gamma_{\mu \nu}^{(2 A)}$ we then obtain

$$
\begin{aligned}
\Gamma_{\mu \nu}^{(2 A)}=\frac{g_{Z}^{2}}{M_{Z}^{2}} \gamma^{\lambda} L & \sum_{f=e, p, n} \int \frac{d^{4} p}{(2 \pi)^{3}} \operatorname{Tr}\left[\gamma_{\lambda}\left(X_{f}+Y_{f} \gamma_{5}\right) A_{\mu \nu}\right] \\
& \times\left(\frac{\delta\left(p^{2}-m_{f}^{2}\right) \eta_{f}(p)}{p^{\prime 2}-m_{f}^{2}}+\frac{\delta\left(p^{\prime 2}-m_{f}^{2}\right) \eta_{f}\left(p^{\prime}\right)}{p^{2}-m_{f}^{2}}\right),
\end{aligned}
$$


where

$$
A_{\mu \nu}^{(f)}=\left(\not p^{\prime}+m_{f}\right) V_{\mu \nu}^{(f)}\left(p, p^{\prime}\right)\left(\not p+m_{f}\right)
$$

Using for $V_{\mu \nu}^{(f)}$ the expression given in Eq. (2.18), we have

$$
A_{\mu \nu}^{(f)}=a_{\mu \nu}^{(f)}-\frac{1}{2} \eta_{\mu \nu}\left[\left(\not p^{\prime}+m_{f}\right)\left(p^{2}-m_{f}^{2}\right)+\left(\not p+m_{f}\right)\left(p^{\prime 2}-m_{f}^{2}\right)\right]
$$

where

$$
a_{\mu \nu}^{(f)}=\left(\not p^{\prime}+m_{f}\right) \frac{1}{4}\left\{\gamma_{\mu}\left(p+p^{\prime}\right)_{\nu}+\gamma_{\nu}\left(p+p^{\prime}\right)_{\mu}\right\}\left(\not p+m_{f}\right) .
$$

When Eq. (3.11) is substituted in Eq. (3.9), the terms that involve the factor of $\eta_{\mu \nu}$ are reduced very simply, and the result can be expressed in the form

$$
\Gamma_{\mu \nu}^{(2 A)}=\Lambda_{\mu \nu}^{(Z)}-b^{(Z)} \psi L \eta_{\mu \nu}
$$

where $\Lambda_{\mu \nu}^{(Z)}$ is given by the same expression given in Eq. (3.9) but with the replacement $A_{\mu \nu} \rightarrow a_{\mu \nu}$, and

$$
b^{(Z)}=\sum_{f} X_{f} b_{f},
$$

with

$$
b_{f}=4 \sqrt{2} G_{F} \int \frac{d^{4} p}{(2 \pi)^{3}} \delta\left(p^{2}-m_{f}^{2}\right) \eta_{f}(p) p \cdot v .
$$

In arriving at Eq. (3.13) we have used the formula

$$
4 \sqrt{2} G_{F} \int \frac{d^{4} p}{(2 \pi)^{3}} \delta\left(p^{2}-m_{f}^{2}\right) \eta_{f}(p) p_{\mu}=b_{f} v_{\mu}
$$

and the relation $\sqrt{2} G_{F}=g_{Z}^{2} / M_{Z}^{2}$.

It is convenient to introduce the particle and antiparticle momentum distribution functions

$$
f_{f, \bar{f}}\left(p_{f}\right)=\frac{1}{e^{\beta\left(p_{f} \cdot v \mp \mu_{f}\right)}},
$$

where the upper and the lower signs hold for the particle and the antiparticle respectively, and

$$
p_{f}^{\mu}=\left(E_{f}, \boldsymbol{P}\right), \quad E_{f}=\sqrt{\boldsymbol{P}^{2}+m_{f}^{2}} .
$$

The corresponding total number densities are given by

$$
n_{f}=2 \int \frac{d^{3} P}{(2 \pi)^{3}} f_{f}, \quad n_{\bar{f}}=2 \int \frac{d^{3} P}{(2 \pi)^{3}} f_{\bar{f}},
$$

and in terms of them Eq. (3.15) can then be written in the form

$$
b_{f}=\sqrt{2} G_{F}\left(n_{f}+n_{\bar{f}}\right) .
$$

The expression for $\Lambda_{\mu \nu}^{(Z)}$ can be simplified by taking the traces and making change of integration variable $p \rightarrow p+q$ in the term containing the factor of $\eta_{f}\left(p^{\prime}\right)$. In this way we obtain

$$
\begin{aligned}
\Lambda_{\mu \nu}^{(Z)}=\frac{g_{Z}^{2}}{M_{Z}^{2}} & \left(\gamma^{\lambda} L\right) \sum_{f} \int \frac{d^{4} p}{(2 \pi)^{3}} \delta\left(p^{2}-m_{e}^{2}\right) \eta_{f}(p) \\
& \times\left\{\frac{X_{f} N_{\mu \nu \lambda}^{(1)}(p, q)-Y_{f} N_{\mu \nu \lambda}^{(2)}(p, q)}{q^{2}-2 p \cdot q}+\frac{X_{f} N_{\mu \nu \lambda}^{(1)}(p,-q)+Y_{f} N_{\mu \nu \lambda}^{(2)}(p,-q)}{q^{2}+2 p \cdot q}\right\}
\end{aligned}
$$


where

$$
\begin{aligned}
& N_{\mu \nu \lambda}^{(1)}(p, q) \equiv(2 p-q)_{\mu}\left[2 p_{\nu} p_{\lambda}-\left(p_{\lambda} q_{\nu}+q_{\lambda} p_{\nu}\right)+(p \cdot q) \eta_{\lambda \nu}\right]+(\mu \leftrightarrow \nu) \\
& N_{\mu \nu \lambda}^{(2)}(p, q) \equiv(2 p-q)_{\mu} i \epsilon_{\nu \lambda \rho \sigma} q^{\rho} p^{\sigma}+(\mu \leftrightarrow \nu) .
\end{aligned}
$$

It is useful to observe that these quantities satisfy the relations

$$
N_{\mu \nu \lambda}^{(1,2)}(-p,-q)=-N_{\mu \nu \lambda}^{(1,2)}(p, q)
$$

from which other similar relations can be obtained. Using them, after performing the $p^{0}$ integration in Eq. (3.21) we obtain finally

$$
\begin{aligned}
\Lambda_{\mu \nu}^{(Z)}=\frac{g_{Z}^{2}}{M_{Z}^{2}}\left(\gamma^{\lambda} L\right) \sum_{f} \int \frac{d^{3} P}{2 E_{f}(2 \pi)^{3}} & \left\{X_{f}\left(f_{f}-f_{\bar{f}}\right)\left[\frac{N_{\mu \nu \lambda}^{(1)}\left(p_{f}, q\right)}{q^{2}-2 p_{f} \cdot q}+(q \rightarrow-q)\right]\right. \\
& \left.-Y_{f}\left(f_{f}+f_{\bar{f}}\right)\left[\frac{N_{\mu \nu \lambda}^{(2)}\left(p_{f}, q\right)}{q^{2}-2 p_{f} \cdot q}-(q \rightarrow-q)\right]\right\} .
\end{aligned}
$$

Further reduction of Eq. (3.24) is not possible without making some assumption about the conditions of the electron gas, in order to be able to carry out the required integrations. Before turning to that, we consider the contributions from the other diagrams, that must be added to Eq. (3.13) to yield the full one-loop result for the induced vertex function.

\subsubsection{Diagram (B)}

Because this diagram contains two $Z$-boson propagators, we retain only the terms proportional to $M_{Z}^{2}$ from the $Z Z h$ vertex given in Eq. (2.36), since the rest will yield results proportional to $1 / M_{Z}^{4}$. For the same reason, we also neglect the momentum dependence of the two $Z$ propagators in the diagram. In this way we then obtain

$$
-i \kappa \Gamma_{\mu \nu}^{(2 B)}=-\left(-i g_{Z}\right)^{2}\left(\frac{i}{M_{Z}^{2}}\right)^{2}\left(i \kappa M_{Z}^{2} a_{\mu \nu \lambda \rho}^{\prime}\right) \gamma^{\lambda} L \sum_{f} \int \frac{d^{4} p}{(2 \pi)^{4}} \operatorname{Tr}\left[\gamma^{\rho}\left(X_{f}+Y_{f} \gamma_{5}\right) i S_{F}^{(f)}(p)\right]
$$

for the contribution to the vertex function. For the background-dependent part, this yields

$$
\Gamma_{\mu \nu}^{\prime(2 B)}=-\frac{g_{Z}^{2}}{M_{Z}^{2}} a_{\mu \nu \lambda \rho}^{\prime} \gamma^{\lambda} L \sum_{f} \int \frac{d^{4} p}{(2 \pi)^{3}} \operatorname{Tr}\left[\gamma^{\rho}\left(X_{f}+Y_{f} \gamma_{5}\right)\left(\not p+m_{f}\right)\right] \delta\left(p^{2}-m_{f}^{2}\right) \eta_{f}(p)
$$

which, by taking the trace and using Eqs. (3.14) and (3.16), can be reduced to

$$
\begin{aligned}
\Gamma_{\mu \nu}^{\prime(2 B)} & =-b^{(Z)} a_{\mu \nu \lambda \rho}^{\prime} \gamma^{\lambda} L v^{\rho} \\
& =-b^{(Z)}\left[\eta_{\mu \nu} \psi+\gamma_{\mu} v_{\nu}+\gamma_{\nu} v_{\mu}\right] L
\end{aligned}
$$

\subsubsection{Diagrams (C) and (D)}

For these diagrams we use the vertices given in Eqs. (2.31) and (2.35). The $O\left(1 / M_{Z}^{2}\right)$ term is given by

$$
-i \kappa \Gamma_{\mu \nu}^{(2 C)}=-\left(-i g_{Z}\right)\left(\frac{i}{M_{Z}^{2}}\right)\left(-i \kappa g_{Z} a_{\mu \nu \lambda \rho}\right) \gamma^{\lambda} L \sum_{f} \int \frac{d^{4} p}{(2 \pi)^{4}} \operatorname{Tr}\left[\gamma^{\rho}\left(X_{f}+Y_{f} \gamma_{5}\right) i S_{F}^{(f)}(p)\right],
$$

and, since $a_{\mu \nu \lambda \rho}$ is symmetric in the indices $\lambda$ and $\rho$, it follows that $\Gamma_{\mu \nu}^{(2 D)}=\Gamma_{\mu \nu}^{(2 C)}$. Following the same steps that led to Eq. (3.27) we find for the background dependent part

$$
\Gamma_{\mu \nu}^{(2 C)}=\Gamma_{\mu \nu}^{(2 D)}=b^{(Z)}\left[\eta_{\mu \nu} \psi+\frac{1}{2}\left(\gamma_{\mu} v_{\nu}+\gamma_{\nu} v_{\mu}\right)\right] L
$$




\subsection{W-exchange diagrams}

\subsubsection{Diagram (A)}

We now consider the diagrams shown in Fig. 1. We begin with diagram A, which corresponds to the expression

$$
-i \kappa \Gamma_{\mu \nu}^{(1 A)}=\left(-\frac{i g}{\sqrt{2}}\right)^{2}(-i \kappa)\left(\frac{i}{M_{W}^{2}}\right) \int \frac{d^{4} p}{(2 \pi)^{4}} \gamma_{\lambda} L i S_{F}^{(e)}\left(p^{\prime}\right) V_{\mu \nu}\left(p, p^{\prime}\right) i S_{F}^{(e)}(p) \gamma^{\lambda} L
$$

where we have neglected the momentum dependence of the $W$ propagator. Using the identity

$$
\left(\gamma^{\lambda} L\right) M\left(\gamma_{\lambda} L\right)=-\left(\gamma^{\lambda} L\right) \operatorname{Tr}\left(M \gamma_{\lambda} L\right)
$$

which is valid for any $4 \times 4$ matrix $M$, Eq. (3.30) can be written as

$$
\Gamma_{\mu \nu}^{(1 A)}=\left(\frac{i g^{2}}{2 M_{W}^{2}}\right) \gamma^{\lambda} L \int \frac{d^{4} p}{(2 \pi)^{4}} \operatorname{Tr}\left[i S_{F}^{(e)}\left(p^{\prime}\right) V_{\mu \nu}\left(p, p^{\prime}\right) i S_{F}^{(e)}(p) \gamma_{\lambda} L\right] .
$$

This expression coincides with what is obtained from Eq. (3.4) by discarding the nucleon terms and then making there the substitutions

$$
\frac{g_{Z}^{2}}{M_{Z}^{2}} \rightarrow \frac{g^{2}}{2 M_{W}^{2}}, \quad X_{e} \rightarrow \frac{1}{2}, \quad Y_{e} \rightarrow-\frac{1}{2}
$$

With this observation, the result for the background-induced part of $\Gamma_{\mu \nu}^{(1 A)}$ can be immediately deduced to be

$$
\Gamma_{\mu \nu}^{\prime(1 A)}=\Lambda_{\mu \nu}^{(W)}-b_{e} \psi L \eta_{\mu \nu}
$$

where

$$
\begin{aligned}
& \Lambda_{\mu \nu}^{(W)}=\frac{g^{2}}{4 M_{W}^{2}}\left(\gamma^{\lambda} L\right) \int \frac{d^{3} P}{2 E_{e}(2 \pi)^{3}}\left\{\left(f_{e}-f_{\bar{e}}\right)\left[\frac{N_{\mu \nu \lambda}^{(1)}\left(p_{e}, q\right)}{q^{2}-2 p_{e} \cdot q}+(q \rightarrow-q)\right]\right. \\
& \left.+\left(f_{e}+f_{\bar{e}}\right)\left[\frac{N_{\mu \nu \lambda}^{(2)}\left(p_{e}, q\right)}{q^{2}-2 p_{e} \cdot q}-(q \rightarrow-q)\right]\right\} .
\end{aligned}
$$

\subsubsection{Other diagrams}

The steps to follow in the calculation of the other diagrams of Fig. 1 are very similar to those for the corresponding diagrams of Fig. 2. Omitting the details here, the background-dependent terms are given by the formulas

$$
\begin{aligned}
-i \kappa \Gamma_{\mu \nu}^{\prime(1 B)} & =-\left(-\frac{i g}{\sqrt{2}}\right)^{2}\left(\frac{i}{M_{W}^{2}}\right)^{2}\left(i \kappa M_{W}^{2} a_{\mu \nu \alpha \beta}^{\prime}\right) \int \frac{d^{4} p}{(2 \pi)^{3}} \delta\left(p^{2}-m_{e}^{2}\right) \eta_{e}(p) \gamma^{\alpha} L\left(\not p+m_{e}\right) \gamma^{\beta} L \\
-i \kappa \Gamma_{\mu \nu}^{\prime(C)} & =-\left(-\frac{i g}{\sqrt{2}}\right)^{2}\left(\frac{i}{M_{W}^{2}}\right)\left(-i \kappa a_{\mu \nu \alpha \beta}\right) \int \frac{d^{4} p}{(2 \pi)^{3}} \delta\left(p^{2}-m_{e}^{2}\right) \eta_{e}(p) \gamma^{\alpha} L\left(\not p+m_{e}\right) \gamma^{\beta} L,
\end{aligned}
$$

with $\Gamma_{\mu \nu}^{\prime(D)}=\Gamma_{\mu \nu}^{\prime(C)}$. Using Eq. (3.16), after some straightforward algebra these reduce to

$$
\begin{aligned}
\Gamma_{\mu \nu}^{(1 B)} & =b_{e}\left[-\eta_{\mu \nu} \psi+\frac{1}{2}\left(\gamma_{\mu} \psi \gamma_{\nu}+\gamma_{\nu} \psi \gamma_{\mu}\right)\right] L \\
\Gamma_{\mu \nu}^{(1 C)}=\Gamma_{\mu \nu}^{(1 D)} & =\frac{1}{2} b_{e}\left[2 \eta_{\mu \nu} \psi-\frac{1}{2}\left(\gamma_{\mu} \psi \gamma_{\nu}+\gamma_{\nu} \psi \gamma_{\mu}\right)\right] L .
\end{aligned}
$$




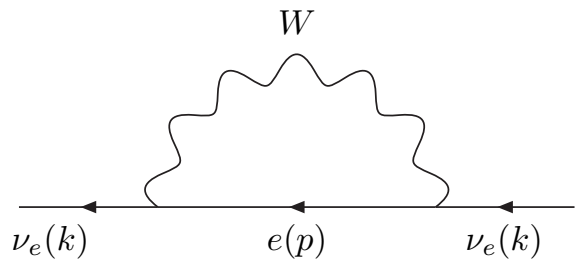

(A)

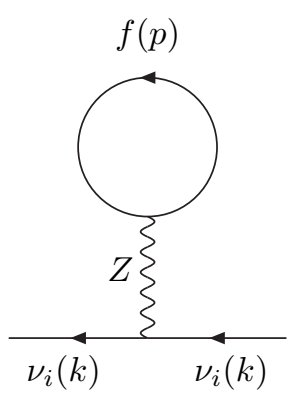

(B)

Figure 3: One-loop diagrams for the self-energy of neutrinos in a medium. Diagram (A) contributes only to $\nu_{e}$, while diagram (B) contributes equally to $\nu_{e}, \nu_{\mu}$ and $\nu_{\tau}$.

\subsection{Complete vertex}

We have completed the calculation of the diagrams that give rise to the background-induced part of the proper vertex of neutrinos with the graviton. The complete vertex function defined in Eq. (3.1) is obtained by adding these individual contributions. For the diagrams of Fig. 1 1 the results are given in Eqs. (3.34) and (3.37), and adding them we obtain

$$
\Gamma_{\mu \nu}^{\prime(1 A)}+\Gamma_{\mu \nu}^{\prime(1 B)}+\Gamma_{\mu \nu}^{(1 C)}+\Gamma_{\mu \nu}^{\prime(1 D)}=\Lambda_{\mu \nu}^{(W)} .
$$

For the diagrams of Fig. 2, the sum of the individual results given in Eqs. (3.13), (3.27) and (3.29) yields

$$
\Gamma_{\mu \nu}^{\prime(2 A)}+\Gamma_{\mu \nu}^{\prime(2 B)}+\Gamma_{\mu \nu}^{\prime(2 C)}+\Gamma_{\mu \nu}^{\prime(2 D)}=\Lambda_{\mu \nu}^{(Z)}
$$

As we have already mentioned, $\Lambda_{\mu \nu}^{(W)}$ contributes to the vertex only when the neutrino is $\nu_{e}$, while $\Lambda_{\mu \nu}^{(W)}$ contributes equally for all the weak-doublet neutrinos, including the $\nu_{e}$. In addition, the vertex function has the tree-level term which, for chiral, left-handed neutrinos is given in Eq. (2.18).

In this way we find that the complete effective vertex function for the various neutrino flavors is given by

$$
\Gamma_{\mu \nu}^{(\nu)}=V_{\mu \nu}^{(\nu)}+\Lambda_{\mu \nu}
$$

where

$$
\Lambda_{\mu \nu}= \begin{cases}\Lambda_{\mu \nu}^{(W)}+\Lambda_{\mu \nu}^{(Z)} & \text { for } \nu_{e} \\ \Lambda_{\mu \nu}^{(Z)} & \text { for } \nu_{\mu}, \nu_{\tau}\end{cases}
$$

Notice that the vertex function is symmetric in the indices $\mu, \nu$, as it should be.

\subsection{Transversality of the vertex}

Before proceeding to the explicit calculations of the neutrino vertex using the one-loop formula given above, it is useful to check that the complete effective vertex satisfies the transversality condition

$$
q^{\mu} \bar{u}_{L}\left(k^{\prime}\right) \Gamma_{\mu \nu}^{(\nu)}\left(k, k^{\prime}\right) u_{L}(k)=q^{\nu} \bar{u}_{L}\left(k^{\prime}\right) \Gamma_{\mu \nu}^{(\nu)}\left(k, k^{\prime}\right) u_{L}(k)=0 .
$$

Here,

$$
\begin{aligned}
k^{\mu} & =\left(\omega_{K}, \boldsymbol{K}\right) \\
k^{\prime \mu} & =\left(\omega_{K^{\prime}}, \boldsymbol{K}^{\prime}\right),
\end{aligned}
$$


where $\omega_{K}$ is the correct dispersion relation for a neutrino mode propagating with momentum $\boldsymbol{K}$ in the medium, and $u_{L}$ is the corresponding spinor. As shown several years ago [1, 5, 6], the spinor $u_{L}(k)$ and the corresponding dispersion relation are found by solving the effective Dirac equation

$$
\left(\not k-\Sigma_{\text {eff }}\right) u_{L}(k)=0,
$$

where $\Sigma_{\text {eff }}$ is the neutrino self-energy in the medium. The chirality of the neutrino interactions dictate that $\Sigma_{\text {eff }}$ has the form

$$
\Sigma_{\text {eff }}=(a \not k+b \psi) L,
$$

where, in general, $a$ and $b$ are functions of $\omega_{K}$ and $K$. In this form, the dispersion relations implied by Eq. (3.44) are given by

$$
\begin{aligned}
& \omega_{K}=K+\frac{b\left(\omega_{K}, K\right)}{1-a\left(\omega_{K}, K\right)} \\
& \bar{\omega}_{K}=K-\frac{b\left(-\bar{\omega}_{K}, K\right)}{1-a\left(-\bar{\omega}_{K}, K\right)},
\end{aligned}
$$

with $\omega_{K}$ corresponding to the neutrino and $\bar{\omega}_{K}$ to the antineutrino, which in the general case must be considered as implicit equations that must be solved for $\omega_{K}$ and $\bar{\omega}_{K}$ as functions of $K$. In the context of our perturbative approach, the solutions to Eq. (3.46) are given approximately by

$$
\begin{aligned}
& \omega_{K}=K+[1+a(K, K)] b(K, K) \\
& \bar{\omega}_{K}=K-[1+a(-K, K)] b(-K, K) .
\end{aligned}
$$

At the one-loop level, the neutrino self-energy in the presence of matter is determined by calculating the diagrams shown in Fig. 3, which has been carried out in detail in the references cited. The result is that, to order $1 / M_{W}^{2}$, the parameter $a$ vanishes while

$$
b_{\text {mat }}= \begin{cases}b_{e}+b^{(Z)} & \text { for } \nu_{e} \\ b^{(Z)} & \text { for } \nu_{\mu}, \nu_{\tau}\end{cases}
$$

where $b_{e}$ and $b^{(Z)}$ are given in Eqs. (3.15) and (3.14), respectively. In this case $\omega_{K}=K+b_{\text {mat }}$ for the neutrinos, and it follows that the spinors satisfy the equation

$$
\left(\not k-b_{\text {mat }} \psi\right) L u_{L}(k)=0
$$

and the relation

$$
k^{2}=2 b_{\mathrm{mat}} k \cdot v-b^{2}
$$

holds. These, together with the analogous relations for $u\left(k^{\prime}\right)$ and $k^{\prime 2}$, imply the useful formulas

$$
\begin{aligned}
\bar{u}_{L}\left(k^{\prime}\right) q u_{L}(k) & =0 \\
k^{2}-k^{\prime 2} & =2 b_{\mathrm{mat}} q \cdot v .
\end{aligned}
$$

We are now in the position to consider the transversality property of the effective vertex. From Eq. 3.22) it follows that

$$
\begin{aligned}
q^{\mu} N_{\mu \nu \alpha}^{(1)} & =\left(2 p \cdot q-q^{2}\right)\left[4 p_{\nu} p_{\alpha}-2 q_{\nu} p_{\alpha}-p_{\nu} q_{\alpha}+p \cdot q \eta_{\nu \alpha}\right] \\
q^{\mu} N_{\mu \nu \alpha}^{(2)} & =\left(2 p \cdot q-q^{2}\right) i \epsilon_{\nu \alpha \lambda \rho} q^{\lambda} p^{\rho}
\end{aligned}
$$

Then from Eq. (3.35)

$$
\begin{aligned}
q^{\mu} \Lambda_{\mu \nu}^{(W)} & =\frac{g^{2}}{2 M_{W}^{2}}\left(\gamma^{\alpha} L\right) \int \frac{d^{4} p}{(2 \pi)^{3}} \delta\left(p^{2}-m_{e}^{2}\right) \eta_{e}(p)\left[2 q_{\nu} p_{\alpha}+p_{\nu} q_{\alpha}-p \cdot q \eta_{\nu \alpha}\right] \\
& =\frac{1}{2} b_{e}\left[2 q_{\nu} \psi+v_{\nu} \not-v \cdot q \gamma_{\nu}\right] L
\end{aligned}
$$


and similarly

$$
q^{\mu} \Lambda_{\mu \nu}^{(Z)}=\frac{1}{2} b^{(Z)}\left[2 q_{\nu} \psi+v_{\nu} \not q-v \cdot q \gamma_{\nu}\right] L
$$

On the other hand, from Eq. (2.19), we obtain

$$
q^{\mu} V_{\mu \nu}^{(\nu)}=\frac{1}{4}\left[\not\left(k+k^{\prime}\right)_{\nu}+\left(k^{2}-k^{\prime 2}\right) \gamma_{\nu}\right] L-\frac{1}{2} q_{\nu}\left[\not k+\not k^{\prime}\right] L .
$$

From these, and using the relations given in Eqs. (3.49) and (3.51), it is easy to verify that the transversality condition stated in Eq. (3.42) is indeed satisfied.

\section{Neutrino index of refraction in a gravitational field}

\subsection{The self-energy of neutrinos in a static gravitational field}

Our aim in this section is to determine the correction to the neutrino index of refraction in the presence of a static gravitational field. To this end, let us consider the scattering of a neutrino by a static gravitational potential $\phi^{\text {ext }}(\boldsymbol{x})$ which is produced by a static mass density $\rho^{\text {ext }}(\boldsymbol{x})$. Defining the Fourier transform of $\phi^{\text {ext }}$ by

$$
\phi^{\operatorname{ext}}(\boldsymbol{x})=\int \frac{d^{3} q^{\prime}}{(2 \pi)^{3}} \phi\left(\boldsymbol{q}^{\prime}\right) e^{i \boldsymbol{q}^{\prime} \cdot \boldsymbol{x}}
$$

with a similar definition for $\rho\left(\boldsymbol{q}^{\prime}\right)$, the corresponding metric is such that, in momentum space,

$$
h^{\mu \nu}\left(\boldsymbol{q}^{\prime}\right)=\frac{1}{\kappa} \phi\left(\boldsymbol{q}^{\prime}\right)\left(2 v^{\mu} v^{\nu}-\eta^{\mu \nu}\right),
$$

where we have used $-2 \boldsymbol{q}^{2} \phi=\kappa^{2} \rho$. The formula in Eq. (4.2) is the solution to the linearized field equation for the metric with the static energy momentum tensor $T^{\mu \nu}=v^{\mu} v^{\nu} \rho^{\text {ext }}$. Under the influence of such an external potential, the off-shell $\nu$ - $\nu$ transition amplitude is then

$$
S_{\nu \nu}=-i \kappa(2 \pi) \delta\left(k^{0}-k^{\prime 0}\right)\left(V_{\mu \nu}^{(\nu)}\left(k, k^{\prime}\right)+\Lambda_{\mu \nu}(0, \mathcal{Q})\right) h^{\mu \nu}\left(\boldsymbol{k}^{\prime}-\boldsymbol{k}\right) .
$$

In Eq. (4.3) we have indicated explicitly the fact that the background part $\Lambda_{\mu \nu}(\Omega, \mathcal{Q})$ of the vertex function does not depend on $k$ and $k^{\prime}$ separately, but only on the variables $\Omega$ and $\mathcal{Q}$ that are defined by writing

$$
q^{\mu}=\left(k-k^{\prime}\right)^{\mu}=(\Omega, \mathcal{Q})
$$

in the rest frame of the medium. Moreover, we have set $\Omega=0$ as implied by the delta function in the right-hand side. In addition, we now restrict ourselves to a uniform field in space, which means that can write

$$
\phi\left(\boldsymbol{k}^{\prime}-\boldsymbol{k}\right)=(2 \pi)^{3} \delta^{(3)}\left(\boldsymbol{k}^{\prime}-\boldsymbol{k}\right) \phi^{\mathrm{ext}}
$$

The justification for this is the usual one. Namely, we assume that we are working in a region of space that is microscopically large but macroscopically small, and therefore the external field is approximately constant over it. The macroscopic dependence of the external field on $\boldsymbol{x}$ can then be restored at the end.

Thus, substituting Eq. (4.5) into Eq. (4.3), we obtain

$$
S_{\nu \nu}=-i(2 \pi)^{4} \delta^{(4)}\left(k-k^{\prime}\right)\left(V_{\mu \nu}^{(\nu)}(k, k)+\Lambda_{\mu \nu}(0, \mathcal{Q} \rightarrow 0)\right)\left(2 v^{\mu} v^{\nu}-\eta^{\mu \nu}\right) \phi^{\mathrm{ext}}
$$

Identifying the gravitational contribution to the self-energy by writing

$$
S_{\nu \nu}=-i(2 \pi)^{4} \delta^{(4)}\left(k-k^{\prime}\right) \Sigma_{G}(k),
$$

we then find

$$
\Sigma_{G}=\Sigma_{g}+\Sigma_{G}^{\prime},
$$


where

$$
\begin{aligned}
& \Sigma_{g}(k)=\phi^{\text {ext }} V_{\mu \nu}^{(\nu)}(k, k)\left(2 v^{\mu} v^{\nu}-\eta^{\mu \nu}\right) \\
& \Sigma_{G}^{\prime}(k)=\phi^{\operatorname{ext}} \Lambda_{\mu \nu}(0, \mathcal{Q} \rightarrow 0)\left(2 v^{\mu} v^{\nu}-\eta^{\mu \nu}\right) .
\end{aligned}
$$

As indicated in Eq. (4.9), the gravitational contribution to the self-energy has two parts. We consider first $\Sigma_{g}(k)$, which is the contribution from the gravitational field when there is no background medium present. Using the expression for $V_{\mu \nu}$ given in Eq. (2.19), we obtain

$$
\Sigma_{g}(k)=\phi^{\mathrm{ext}}(\not k+2 k \cdot v \psi) L,
$$

which in terms of the parametrization of the self-energy given in Eq. $(3.45)$ amounts to

$$
a_{g}=\phi^{\mathrm{ext}}, \quad b_{g}=\phi^{\mathrm{ext}} 2 k \cdot v .
$$

\subsection{The matter-induced gravitational contribution to the self energy}

Since the expression for $\Lambda_{\mu \nu}$ contains the factor $\gamma^{\lambda} L$, it is useful to define the vector $t_{\lambda}$ by writing

$$
\left(2 v^{\mu} v^{\nu}-\eta^{\mu \nu}\right) \Lambda_{\mu \nu}(\Omega, \mathcal{Q}) \equiv\left(\gamma^{\lambda} L\right) t_{\lambda}(\Omega, \mathcal{Q})
$$

in terms of which

$$
\Sigma_{G}^{\prime}(k)=\phi^{\text {ext }} \gamma^{\lambda} t_{\lambda}(0, \mathcal{Q} \rightarrow 0) .
$$

In an isotropic background, which we have assumed by writing the distribution functions as in Eq. (3.17), $t_{\alpha}$ can be expressed in the form

$$
t_{\lambda}=A_{G} q_{\lambda}+B_{G} \tilde{v}_{\lambda}
$$

where we have defined

$$
\tilde{v}^{\lambda} \equiv v^{\lambda}-\frac{v \cdot q}{q^{2}} q^{\lambda}
$$

Since $q \cdot \tilde{v}=0$, the scalar functions can be calculated by using

$$
\begin{aligned}
& A_{G}=\frac{1}{q^{2}} q \cdot t, \\
& B_{G}=\frac{1}{\tilde{v}^{2}} \tilde{v} \cdot t .
\end{aligned}
$$

If $A_{G}(0, \mathcal{Q})$ is not singular in the limit $\mathcal{Q} \rightarrow 0$, then Eqs. (4.13) and (4.14) imply that

$$
\Sigma_{G}^{\prime}=\phi^{\text {ext }} B_{G}(0, \mathcal{Q} \rightarrow 0) \psi L,
$$

so that only $B_{G}$ needs to be evaluated. To prove that this is the case, notice from Eq. (3.22) that

$$
v^{\lambda}\left(2 v^{\mu} v^{\nu}-\eta^{\mu \nu}\right) N_{\mu \nu \lambda}^{(2)}=q^{\lambda}\left(2 v^{\mu} v^{\nu}-\eta^{\mu \nu}\right) N_{\mu \nu \lambda}^{(2)}=0 .
$$

Therefore, $A_{G}$ and $B_{G}$ are given by

$$
\begin{aligned}
& A_{G}=\sqrt{2} G_{F} \begin{cases}A_{e}+\sum_{f} X_{f} A_{f} & \text { for } \nu_{e}, \\
\sum_{f} X_{f} A_{f} & \text { for } \nu_{\mu}, \nu_{\tau},\end{cases} \\
& B_{G}=\sqrt{2} G_{F} \begin{cases}B_{e}+\sum_{f} X_{f} B_{f} & \text { for } \nu_{e}, \\
\sum_{f} X_{f} B_{f} & \text { for } \nu_{\mu}, \nu_{\tau},\end{cases}
\end{aligned}
$$


with

$$
\begin{aligned}
& A_{f}=\frac{1}{q^{2}} \int \frac{d^{3} P}{(2 \pi)^{3} 2 E_{f}}\left(f_{f}-f_{\bar{f}}\right)\left[\frac{I_{a}(\Omega, \mathcal{Q})}{q^{2}-2 p_{f} \cdot q}-(q \rightarrow-q)\right], \\
& B_{f}=\frac{1}{\tilde{v}^{2}} \int \frac{d^{3} P}{(2 \pi)^{3} 2 E_{f}}\left(f_{f}-f_{\bar{f}}\right)\left[\frac{I_{b}(\Omega, \mathcal{Q})}{q^{2}-2 p_{f} \cdot q}+(q \rightarrow-q)\right],
\end{aligned}
$$

where we have defined

$$
\begin{aligned}
& I_{a}=q^{\lambda}\left(2 v^{\mu} v^{\nu}-\eta^{\mu \nu}\right) N_{\mu \nu \lambda}^{(1)}, \\
& I_{b}=\tilde{v}^{\lambda}\left(2 v^{\mu} v^{\nu}-\eta^{\mu \nu}\right) N_{\mu \nu \lambda}^{(1)} .
\end{aligned}
$$

From the expression given in Eq. (3.22) for $N_{\mu \nu \lambda}^{(1)}$ it is straightforward to see that

$$
I_{a}(0, \mathcal{Q})=\left(\mathcal{Q}^{2}-2 \boldsymbol{P} \cdot \mathcal{Q}\right)\left(8 E_{f}^{2}-4 m_{f}^{2}-2 \boldsymbol{P} \cdot \mathcal{Q}\right) .
$$

Using this in Eq. (4.20), it follows that $A_{f}(0, \mathcal{Q})$ is proportional to the integral of $\boldsymbol{P} \cdot \mathcal{Q}$, which is zero for an isotropic distribution. Therefore

$$
A_{f}(0, \mathcal{Q})=0,
$$

which proves Eq. (4.17).

To evaluate $B_{f}(0, \mathcal{Q} \rightarrow 0)$, we use Eq. (4.21) and the definition of $N_{\mu \nu \alpha}^{(1)}$ given in Eq. (3.22) to obtain, after rearranging some terms,

$$
I_{b}(0, \mathcal{Q})=2 E_{f}\left(8 E_{f}^{2}-4 m_{f}^{2}-2 \mathcal{Q}^{2}\right)-6 E_{f}\left(2 \boldsymbol{P} \cdot \mathcal{Q}-\mathcal{Q}^{2}\right) .
$$

Using this in Eq. (4.20),

$$
B_{f}(0, \mathcal{Q}) \equiv-3\left(n_{f}-n_{\bar{f}}\right)+\int \frac{d^{3} P}{(2 \pi)^{3}}\left[\frac{F-2 \mathcal{Q}^{2}\left(f_{f}-f_{\bar{f}}\right)}{-\mathcal{Q}^{2}+2 \boldsymbol{P} \cdot \mathcal{Q}}+(\mathcal{Q} \rightarrow-\mathcal{Q})\right],
$$

with

$$
F \equiv 4\left(2 E_{f}^{2}-m_{f}^{2}\right)\left(f_{f}-f_{\bar{f}}\right) .
$$

To evaluate this integral, we make the change of variables $\boldsymbol{P} \rightarrow \boldsymbol{P}+\frac{1}{2} \mathcal{Q}$ in the first term, and $\boldsymbol{P} \rightarrow \boldsymbol{P}-\frac{1}{2} \mathcal{Q}$ in the second one, remembering that the distribution functions and $F$ are functions of $\boldsymbol{P}$. This procedure yields

$$
B_{f}(0, \mathcal{Q} \rightarrow 0)=J_{f}+O\left(\mathcal{Q}^{2}\right)
$$

where

$$
\begin{aligned}
J_{f} & =-3\left(n_{f}-n_{\bar{f}}\right)+\int \frac{d^{3} P}{(2 \pi)^{3}}\left(\frac{\mathcal{Q} \cdot \boldsymbol{\nabla}_{P} F}{2 \boldsymbol{P} \cdot \mathcal{Q}}\right), \\
& =-3\left(n_{f}-n_{\bar{f}}\right)+\int \frac{d^{3} P}{(2 \pi)^{3} 2 E_{f}} \frac{d F}{d E_{f}} .
\end{aligned}
$$

In arriving at Eq. (4.28) we have used the definition in Eq. (3.19), and in writing the second equality we have used the fact the the function $F$ depend on $\boldsymbol{P}$ only through $E_{f}$. From Eqs. (4.19), (4.17) and (4.27) we finally obtain

$$
\Sigma_{G}^{\prime}(k)=b_{G} \psi
$$

where

$$
b_{G}=\phi^{\mathrm{ext}} \sqrt{2} G_{F} \times \begin{cases}J_{e}+\sum_{f} X_{f} J_{f} & \text { for } \nu_{e} \\ \sum_{f} X_{f} J_{f} & \text { for } \nu_{\mu}, \nu_{\tau}\end{cases}
$$




\subsection{Dispersion relations}

We are now in the position to determine the dispersion relation for the neutrinos in the medium in the presence of a static gravitational field. Referring back to Eq. (3.45), the results we have obtained can be summarized by writing

$$
\begin{aligned}
a & =a_{g} \\
b & =b_{\text {mat }}+b_{g}+b_{G} .
\end{aligned}
$$

The terms $a_{g}$ and $b_{g}$, which are given in Eq. (4.11), represent the contribution from pure gravity and are present even in the case that there is no matter. On the other hand, the term $b_{\text {mat }}$, given in Eq. (3.48), arises due to the presence of the background medium, independently of whether a gravitational field is present or not. Finally, $b_{G}$, given in Eq. (4.30), is the contribution that arises due to the simultaneous presence of matter and the external gravitational field. Thus, substituting Eq. (4.31) in Eq. (3.47), and keeping in mind that we are allowed to retain only terms that are linear in the external gravitational potential, we obtain

$$
\omega_{K}=K+2 K \phi^{\mathrm{ext}}+b_{\mathrm{mat}}+\left(\phi^{\mathrm{ext}} b_{\mathrm{mat}}+b_{G}\right) .
$$

The corresponding formula for $\bar{\omega}_{K}$ is obtained from this by reversing the sign in front of $b_{\text {mat }}$ and $b_{G}$. Apart from the first term in the right-hand side Eq. (4.32), which corresponds just to the vacuum dispersion relation, the other terms have the following meaning.

The second term has a purely gravitational origin. If we take it by itself and neglect the effects of the medium, it gives rise to the dispersion relation

$$
\bar{\omega}_{K}=\omega_{K}=K\left(1+2 \phi^{\mathrm{ext}}\right),
$$

which is equivalent to say that the neutrino and antineutrino acquire an index of refraction given by

$$
\mathcal{N}_{0} \equiv 1-2 \phi^{\text {ext }}
$$

This is the same result that is obtained by solving the equation

$$
k_{\mu} k_{\nu} g^{\mu \nu}=0
$$

which is the appropriate equation to solve in order to determine the dispersion relation for the photon or a massless scalar particle. That purely gravitational term also gives rise to effects analogous to the gravitational red shift and bending of light. For example, consider a neutrino beam propagating along the $z$ direction in the vicinity of a massive body of mass $M$ situated at the origin, with an impact parameter $b$. In the eikonal approximation, $\omega_{K}(t)$ and $\boldsymbol{K}(\boldsymbol{x})$ satisfy the Hamilton equation

$$
\frac{\partial \boldsymbol{K}}{\partial t}=-\nabla \omega_{K}
$$

which can be used to obtain the transverse component $K_{x}$ that the momentum develops as a consequence of the influence of the gravitational field. Substituting $\omega_{K}=\left(1+2 \phi^{\text {ext }}\right) K$ in Eq. (4.36), we can write

$$
\frac{K_{x}}{K}=-2 \int_{-\infty}^{\infty} d t \frac{\partial \phi^{\mathrm{ext}}(r)}{\partial b}
$$

where the position vector is $\boldsymbol{r}=(b, 0, t)$ and $\phi^{\operatorname{ext}}(r)=-G M / r$. From this we obtain the bending formula for neutrinos

$$
\frac{K_{x}}{K}=-\frac{4 G M}{b},
$$

which is the same result as the corresponding formula for photons.

In contrast to the purely gravitational terms, the matter-dependent terms contribute with opposite signs to the neutrino and antineutrino indices of refraction. The term $b_{\text {mat }}$ on the right side of Eq. (4.32), which denotes the matter contribution in the absence of a gravitational field, is the usual Wolfenstein term. It is flavor dependent, and it is the origin of the MSW mechanism for neutrino oscillations in matter. The 
remaining terms are the new contributions we set out to determine. One of them is exactly the Wolfenstein term multiplied by the external gravitational potential. Since we are considering weak gravitational fields, that term is much smaller than the usual Wolfenstein term and can be neglected. Thus, the dispersion relation reduces to

$$
\omega_{K}=K+2 \phi^{\mathrm{ext}} K+b_{\mathrm{mat}}+b_{G} .
$$

which can be equivalently stated in terms of the index of refraction

$$
\begin{aligned}
\mathcal{N}_{\nu} & \equiv \frac{K}{\omega_{K}} \\
& =\mathcal{N}_{0}-\frac{b_{\text {mat }}}{K}-\frac{b_{G}}{K} .
\end{aligned}
$$

The term that we have denoted by $b_{G}$ is more interesting since it can have a non-trivial dependence on the temperature and density of the background material. It is flavor dependent as well, and it arises because of the presence of both matter and gravitational fields. In order to be able to consider its possible physical effects, in the ensuing section we will estimate its magnitude for various physical conditions of the background medium.

\subsection{Estimates of the matter-gravitational effects}

The question we wish to address here is how large are the matter-gravitational effects, represented by the term $b_{G}$ in Eq. (4.40), relative to the Wolfenstein term $b_{\text {mat }}$. To answer this question we need an estimate of the integral $H_{f}$, which we have carried out in the Appendix, and also make some assumptions about the composition of the background for the possible physical situations of interest.

As an example, let us consider a medium such as the Sun or a supernova. In these, the conditions are such that the nucleons are non-relativistic. Therefore, using the results given in Eqs. (A.4) and (A.8), we can write for the nucleons $(N=p, n)$

$$
J_{N}= \begin{cases}-\beta m_{N} n_{N} & \text { classical nucleon gas } \\ -\frac{3 n_{N}}{v_{F N}^{2}} & \text { degenerate nucleon gas }\end{cases}
$$

where $v_{F N}$ stands for the Fermi velocity of the nucleon gas.

Regarding the electrons, if they are relativistic then from Eq. (A.11) we have $J_{e}=-5 n_{e}$. In this case, the electron contribution in Eq. (4.30) is of the order of the Wolfenstein term multiplied by $\phi^{\text {ext }}$, and therefore it is unimportant. On the other hand, this is not necessarily the case for a non-relativistic electron gas. In analogy with Eq. (4.41) for this case

$$
J_{e}= \begin{cases}-\beta m_{e} n_{e} & \text { classical non-relativistic electron gas }, \\ -\frac{3 n_{e}}{v_{F e}^{2}} & \text { degenerate non-relativistic electron gas } .\end{cases}
$$

To assess further the possible importance of the matter-gravitational contributions to the index of refraction, we also need some knowledge of the magnitude of the gravitational potential that could be involved. If we take as a guiding value the potential at the surface of the Sun,

$$
\phi_{\odot}=-2 \times 10^{-6},
$$

we find that the matter-gravitational contributions could be relevant under the appropriate conditions. This is particularly true for the nucleon terms, which have the enhancement factors $\beta m_{N}$ or $v_{F N}^{-2}$ in the classical and the degenerate cases, respectively. This is not unexpected, since the gravitational potential couples more strongly to the more massive particles.

As we have already mentioned, and as Eq. (4.30) clearly indicates, the nucleon contributions to $b_{G}$ are the same for all the neutrino flavors, and therefore they are irrelevant for neutrino oscillations involving only weak-doublet neutrinos. On the other hand, they are relevant for oscillation phenomena in which sterile neutrinos participate and, as we have shown above, they may be important. Furthermore, $b_{\text {mat }}$ and $b_{G}$ have a different dependence on the neutrino coordinate as it propagates through the medium, a property that may have also distinctive implications. 


\section{Conclusions}

In this work we have determined the effects of a static gravitational potential on the neutrino index of refraction in matter. This has been done by first carrying out the one-loop calculation of the matter-induced gravitational couplings of the neutrinos, and then by determining the corrections to the neutrino self-energy that such couplings imply in the presence of the gravitational potential.

As a consistency check of the one-loop formulas, we showed explicitly that the effective gravitational vertex of the neutrino is transverse. This required that the correct dispersion relation and wavefunction associated with the external neutrino modes be used, and not their vacuum counterpart. We emphasize again that it is crucial that, in the calculation of the one-loop diagrams, we have used the full off-shell formula for the tree-level gravitational vertex function of the internal fermions in the loop, and not the on-shell limit that is customarily quoted.

As indicated in Section 4, the matter-gravitational contribution to the neutrino index of refraction could be relevant in the context of matter-enhanced neutrino oscillations, and in particular in phenomena involving the so-called sterile neutrinos. This may occur not only because their magnitude could be non-negligible, but also because they have a different dependence on the coordinate compared with the standard Wolfenstein term. Whether or not these gravitational effects can lead to interesting observable consequences in specific contexts, such as the supernova or the Solar neutrino problem, is an open question that needs further detailed study.

The results presented here indicate that such studies could be well motivated, and our work sets down the arena to carry them out on firm grounds and in a systematic fashion.

Note added: After this paper was submitted for publication, the work by Piriz, Roy and Wudka [16] was brought to our attention, in which the tree-level gravitational coupling of the neutrino is also considered without violating the Equivalence Principle. However, these authors assume an intrinsic magnetic moment of the neutrino, and they concern themselves with the effect of the (vacuum) gravitational interactions on the magnetic spin flip oscillations. They do not consider the effect of matter on the gravitational neutrino interactions, which is the focus of the present work.

\section{ACKNOWLEDGEMENT}

The work of J.F.N. has been partially supported by the US National Science Foundation Grant PHY-9600924.

\section{A Evaluation of $J_{f}$}

We consider the evaluation of the quantity $J_{f}$ defined in Eq. (1.28) for various conditions of the background fermion gas. That expression for $J_{f}$ can be rewritten by taking the derivative of the function $F$ given in Eq. (4.26) and using Eq. (3.19). In this way Eq. (4.28) becomes

$$
J_{f}=\left(n_{f}-n_{\bar{f}}\right)+H_{f}
$$

where

$$
H_{f}=4 \int \frac{d^{3} P}{(2 \pi)^{3}}\left(E_{f}-\frac{m_{f}^{2}}{2 E_{f}}\right) \frac{d}{d E_{f}}\left(f_{f}-f_{\bar{f}}\right) .
$$

In order to carry out this integral, we consider the following illustrative cases.

\section{A.1 Classical non-relativistic gas}

In this case, we can put $f_{\bar{f}} \simeq 0$ and use

$$
\frac{d f}{d E_{f}} \simeq-\beta f
$$

Using these and remembering that we can approximate $E_{f} \simeq m_{f}$ in the integrand, we then obtain from Eqs. (A.1) and (A.2)

$$
J_{f} \simeq H_{f} \simeq-\beta m_{f} n_{f},
$$

where we have used the fact that $\beta m_{f} \gg 1$ for a non-relativistic gas. 


\section{A.2 Degenerate gas at zero temperature}

For this case, the distribution function is given by

$$
f=\Theta\left(E_{F}-E\right), \quad \bar{f} \approx 0,
$$

where $\Theta$ denotes the step function and $E_{F}$ is the Fermi energy. Thus $d f / d E=-\delta\left(E-E_{F}\right)$. Putting this back into Eq. A.2 and using $P_{F}^{3}=3 \pi^{2} n_{f}$ for the Fermi momentum, we obtain

$$
H_{f}=-\left[6 n_{f}+m_{f}^{2}\left(\frac{3 n_{f}}{\pi^{4}}\right)^{1 / 3}\right]
$$

which in turn implies

$$
J_{f}=-\left[5 n_{f}+m_{f}^{2}\left(\frac{3 n_{f}}{\pi^{4}}\right)^{1 / 3}\right] .
$$

No assumption has been made here about whether or not the gas is non-relativistic. However, in the relativistic or non-relativistic limits, this formula reduces to

$$
J_{f}= \begin{cases}-5 n_{f} & \text { relativistic gas } \\ -\frac{3 n_{f}}{v_{F}^{2}} & \text { non-relativistic gas }\end{cases}
$$

where we have used $p_{F}=m_{f} v_{F}$ for the non-relativistic case.

\section{A.3 Ultra-relativistic gas}

In this case we neglect the mass of the background particles and therefore we approximate Eq. (A.2) by

$$
H_{f}=4 \int \frac{d^{3} P}{(2 \pi)^{3}} P \frac{d}{d P}\left(f_{f}-f_{\bar{f}}\right) .
$$

By carrying out a partial integration and using Eq. (3.19) this is equivalent to

$$
H_{f}=-6\left(n_{f}-n_{\bar{f}}\right) \text {, }
$$

and therefore

$$
J_{f}=-5\left(n_{f}-n_{\bar{f}}\right) .
$$

\section{References}

[1] L. Wolfenstein, Phys. Rev. D17, 2369 (1978); P. Langacker, J. P. Leveille and J. Sheiman, Phys. Rev. D27, 1228 (1983); S. P. Mikheyev and A. Yu. Smirnov, Sov. J. Nucl. Phys. 42, 913 (1985).

[2] For a recent review and references to earlier literarure see, for example, Georg G. Raffelt, hep$\mathrm{ph} / 9712292$.

[3] A. Kusenko and G. Segrè, Phys. Rev. Lett. 77, 4872 (1996); Phys. Lett. B396, 197 (1997).

[4] D. Notzold and G. Raffelt, Nucl. Phys. B307, 924 (1988).

[5] P. B. Pal and T. N. Pham, Phys. Rev. D40, 259 (1989).

[6] J. F. Nieves, Phys. Rev. D40, 866 (1989).

[7] J. C. D’Olivo, J. F. Nieves and M. Torres, Phys. Rev. D46, 1172 (1992).

[8] J. C. D'Olivo, José F. Nieves and P. B. Pal, Phys. Rev. D40, 3679 (1989). 
[9] See also, S. Esposito and G. Capone, Z. Phys. C70, 55 (1996);

P. Elmfors, D. Grasso and G. Raffelt, Nucl. Phys. B479, 3 (1996);

A. Yu. Smirnov, in ICHEP'96, Proceedings of the 28th International Conference on High Energy Physics, Warsaw, Poland, 1996 edited by Z. A. Aduk and A. Wroblewski (World Scientific, Singapore, 1997);

J. C. D'Olivo and J. F. Nieves, Phys. Lett. B383, 87 (1996); Phys. Rev. D56, 5898 (1997).

[10] J. C. D’Olivo, J. F. Nieves and P. B. Pal, Phys. Rev. Lett. 64, 1088 (1990); J. F. Nieves and P. B. Pal, Phys. Rev. D56, 365 (1997).

[11] J. F. Nieves and P. B. Pal, Phys. Rev. D39, 652 (1989); ibid.40, 2148E (1989);

S. Mohanty, J. F. Nieves and P. B. Pal, "Optical activity of a neutrino gas", hep-ph/9712414.

[12] M. Gasperini, Phys. Rev. D38, 2635 (1988); A. Halprin, C. N. Leung, Phys. Rev. Lett. 67, 1833 (1991).

[13] D. Boulware and S. Deser, Ann. Phys. 89, 193 (1975). See Eq. (73).

[14] See for example, M. D. Scadron, Advanced Quantum Theory, (Springer-Verlag, New York, 1997).

[15] This notation and that for the vierbein fields should not be the source of confusion, firstly because the vierbeins have two indices while the velocity has only one, and secondly because the vierbeins will not be used in the rest of the paper.

[16] D. Piriz, M. Roy and J. Wudka, Phys. Rev. D56, 1403 (1997). 